\title{
Novel Renal Autologous Cell Therapy for Type 2 Diabetes Mellitus Chronic Diabetic Kidney Disease: Clinical Trial Design
}

\author{
Joseph Stavas ${ }^{a}$ David Gerber ${ }^{b}$ Steven G. Coca ${ }^{c} \quad$ Arnold L. Silva $^{d}$ Ashley Johns ${ }^{a}$ \\ Deepak Jain $^{a}$ Tim Bertram ${ }^{a}$ Maria Díaz-González de Ferris ${ }^{e}$ George Bakris ${ }^{f}$ \\ aProKidney, Raleigh, NC, USA; b Department of Surgery, The University of North Carolina, Chapel Hill, NC, USA; \\ 'Department of Medicine, Icahn School of Medicine at Mt. Sinai, New York, NY, USA; 'Boise Kidney and Hypertension, \\ Boise, ID, USA; ${ }^{\text {DDepartment }}$ of Pediatrics, The University of North Carolina, Chapel Hill, NC, USA; fDepartment of \\ Medicine, The University of Chicago, Chicago, IL, USA
}

\section{Keywords}

Renal autologous cell therapy · Neo-kidney augment ·

Chronic kidney disease - Diabetic nephropathy .

Diabetes-associated chronic kidney disease $\cdot$ Renal outcomes

\begin{abstract}
Background: Cell therapies explore unmet clinical needs of patients with chronic kidney disease with the potential to alter the pathway toward end-stage kidney disease. We describe the design and baseline patient characteristics of a phase II multicenter clinical trial utilizing the novel renal autologous cell therapy (REACT), by direct kidney parenchymal injection via the percutaneous approach in adults with type 2 diabetic kidney disease (T2DKD), to delay or potentially avoid renal replacement therapy. Design: The study conducted a prospective, multicenter, randomized control, open-label, phase II clinical trial between an active treatment group (ATG) receiving REACT from the beginning of the trial and a contemporaneous deferred treatment group (DTG) receiving standard of care for 12 months before crossing over to receive REACT. Objectives: The objective of this study was to establish the safety and efficacy of 2 REACT injections with computed tomography guidance, into the renal cortex of patients with T2DKD administered 6 months apart, and to compare the longitudinal change in renal function between
\end{abstract}

the ATG and the DTG. Setting: This was a multicenter study conducted in major US hospitals. Patients: We enrolled eighty-three adult patients with T2DKD, who have estimated glomerular filtration rates (eGFRs) between 20 and $50 \mathrm{~mL} /$ $\mathrm{min} / 1.73 \mathrm{~m}^{2}$. Methods: All patients undergo an image-guided percutaneous kidney biopsy to obtain epithelial phenotype selective renal cells isolated from the kidney tissue that is then expanded ex vivo over 4-6 weeks, resulting in the REACT biologic product. Patients are randomized 1:1 into the ATG or the DTG. Primary efficacy endpoints for both study groups include eGFR measurements at baseline and at 3-month intervals, through 24 months after the last REACT injection. Safety analyses include biopsy-related complications, REACT injection, and cellular-related adverse events. The study utilizes Good Clinical and Manufacturing Practices and a Data and Safety Monitoring Board. The sample size confers a statistical power of $80 \%$ to detect an eGFR change in the ATG compared to the DTG at 24 months with an $a=$ 0.05. Limitations: Blinding cannot occur due to the intent to treat procedure, biopsy in both groups, and open trial design. Conclusion: This multicenter phase II randomized clinical trial is designed to determine the efficacy and safety of REACT in improving or stabilizing renal function among patients with T2DKD stages 3a-4.

(c) 2022 The Author(s)

Published by S. Karger AG, Basel karger@karger.com www.karger.com/ajn

Karger $\stackrel{\text { ' }}{5}$
(C) 2022 The Author(s)

Published by S. Karger AG, Basel

This is an Open Access article licensed under the Creative Common Attribution-NonCommercial-4.0 International License (CC BY-NC) (http://www.karger.com/Services/OpenAccessLicense), applicable to the online version of the article only. Usage and distribution for commercial purposes requires written permission.
Correspondence to:

Joseph Stavas, joseph.stavas@ prokidney.com 


\section{Introduction}

Cell therapies attempt to address unserved clinical expectations and needs of patients and medical professionals for various chronic medical conditions, including chronic kidney disease (CKD) $[1,2]$. CKD affects about $18 \%$ of the world population (850 million individuals), with up to $50 \%$ secondary to type 2 diabetes which encumbers large cost expenditures, given its risk for progression to end-stage kidney disease [3-5]. Current type 2 diabetic kidney disease (T2DKD) treatments such as renal angiotensin system inhibitors and SGLT2 inhibitors utilize small molecules to modulate biochemical mechanisms of action, albeit in a damaged structural environment [6-8]. In comparison, cell therapies have the potential to induce structural nephro-restoration to stabilize or improve renal function with infrequent dosing and few or no immunologic concerns [9-11].

Most cell-based therapies under investigation for CKD utilize intravascular-delivered mesenchymal cell lines with variable dosing, potency, stability, and end-organ effect. REACT endeavors to address these shortfalls by using a personalized treatment approach with autologous, homologous cells intended to restore nephron structure, improve kidney function, and decrease CKD-related comorbidities. REACT consists of selective renal cells (SRCs) isolated and expanded using Good Manufacturing Process (GMP) from kidney biopsy tissue obtained from CKD patients.

Our preclinical experience with renal autologous cells demonstrated improved renal function and nephron repair, based on surrogate markers and histologic findings in diabetic models [12]. A first-in-human phase I trial in moderate to advanced T2DKD patients confirmed cell safety and stabilization of various renal function surrogate markers during laparoscopic-guided direct renal cortex REACT delivery [13]. We herein report the design of a novel phase II open-label cell therapy trial of REACT, whereby expanded autologous renal cells are percutaneously reinjected into patients with T2DKD stages $3 \mathrm{a}-4$, in a specific locoregional site of the kidney identified with computed tomography (CT) imaging. Our clinical trial design is shown in Figure 1 and is registered at the following website: https://clinicaltrials.gov/ct2/show/NCT02836574.

Our clinical trial objectives are to establish the efficacy and safety of 2 image-guided percutaneous injections of REACT into the renal cortex of patients with T2DKD, administered 6 months apart, and to compare the change in renal function between an active treatment group (ATG) and a contemporaneous deferred treatment group (DTG).

\section{Materials and Methods}

\section{Trial Design}

This is a prospective, multicenter, randomized control, openlabel, phase II clinical trial. Patients were randomized in a 1:1 ratio into 2 groups (ATG $n=42$ or DTG $n=41$ ) after manufacturing confirmed the adequacy/quality of the donor kidney biopsy material and the date of REACT injection. Randomization was performed with the Interactive Web Randomization System. Since this is an open-label study, the study participants, investigators, site staff, and sponsor are not blinded to the treatment assignment. Following a kidney biopsy, patient randomization takes place into the ATG or DTG cohorts. The ATG receives REACT treatment from the beginning of the trial, whereas the DTG will receive SOC for 12 months before crossing over to receive the REACT treatment.

\section{Participants}

We included 83 patients, ages $30-80$ years, who have T2DKD and an established declining estimated glomerular filtration rate (eGFR) between 20 and $50 \mathrm{~mL} / \mathrm{min} / 1.73 \mathrm{~m}^{2}$ (CKD stages $3 \mathrm{a}-4$ ). Patients are from 17 institutions in the USA. To define the rate of CKD progression, baseline renal function was determined by 2 or more eGFR measurements, at least 3 months apart and 18 months pre-intervention. Patient demographics including clinical/laboratory characteristics and concomitant medications are shown in Table 1. The patient's blood pressure must be $<150 / 90 \mathrm{~mm} \mathrm{Hg}$ on stable doses of antihypertensives (defined as dose adjustment to no less than one-half of the current dosage or to no more than 2 times the current dosage), 6 weeks prior to the REACT injection, but dose interruptions for up to 7 days due to medical necessity are allowed. If their antihypertensives included an angiotensin-converting enzyme inhibitor or an angiotensin receptor blocker, treatment must have been initiated at least 8 weeks prior to their renal biopsy. Participants refrain from consuming nonsteroidal anti-inflammatory drugs (including aspirin), fish oil, platelet inhibitors, and anticoagulants 7 days before and after the renal biopsy and REACT injections. Inclusion and exclusion criteria are described in Table 2.

\section{Biopsy and REACT Injection Procedures}

All patients underwent an outpatient percutaneous renal biopsy and then were randomized to either the ATG or the DTG of the trial. ATG patients receive a REACT dose as soon as REACT is manufactured and delivered to the research site, whereas the DTG patients received the intervention after 12 months of SOC treatment. Patients received 2 injections of REACT given 6 months apart. In addition, each patient's rate of renal function change (based on the previous 18 months) serves as a comparator to establish the rate of DKD progression. Furthermore, the DTG patients serve only as contemporaneous comparators to the ATG patients for the 12 months during SOC treatment and against themselves once they cross over to receive REACT. The final statistical analysis will reflect the comparator time line and account for expected progression of CKD in the DTG.

A selective population of renal cells are obtained from the patient's renal cortex via percutaneous kidney biopsy, using a standard-of-clinical-care image-guided method (ultrasound or CT). Two 16-gauge or 4 18-gauge core samples $>1.5 \mathrm{~cm}$ in length are obtained and placed immediately in the transport medium and 


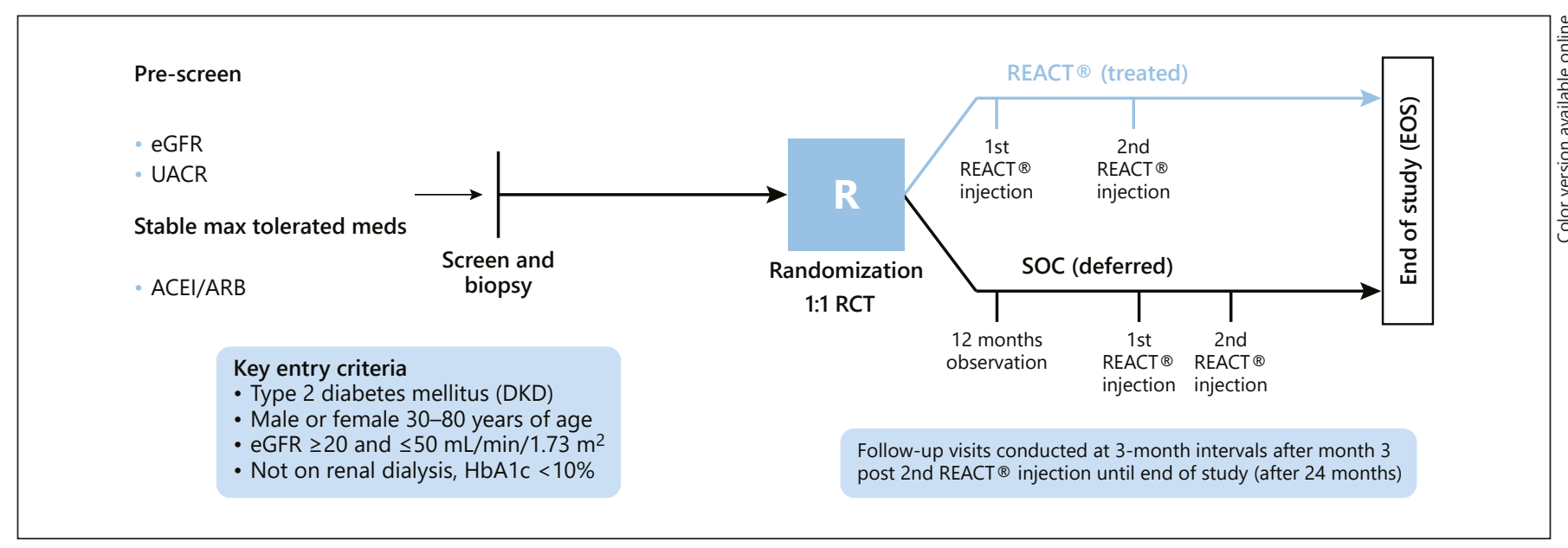

Fig. 1. Trial design outline. R occurs after kidney biopsy into ATG or DTG. The deferred group receives REACT following 12 months of SOC. R, randomization; SOC, standard of care; REACT, Renal Autologous Cell Therapy; ATG, active treatment group; DTG, deferred treatment group; DKD, diabetes kidney disease.

Table 1. Baseline characteristics, laboratory values, and concomitant medications for patients enrolled in the study

\begin{tabular}{llll}
\hline Patient information $(N=83)$ & Mean $(\mathrm{SD}, \%)$ & Concomitant medications & $N(\%)$ \\
\hline Age & $65.2(9.43)$ & ACEi's & $34(41.0)$ \\
Male & $56(67.5)$ & ARB's & $37(44.6)$ \\
Female & $27(32.5)$ & Beta blockers & $42(50.6)$ \\
Ethnicity (Hispanic or Latino) & $11(13.3)$ & Alpha 2 agonists & $9(10.8)$ \\
Ethnicity (non-Hispanic or Latino) & $72(86.7)$ & Diuretics & $51(61.4)$ \\
American Indian & $1(1.2)$ & Loop diuretics & $28(33.7)$ \\
Asian & $1(1.2)$ & Platelet aggregate inhibitors & $50(60.2)$ \\
Black or African American & $7(8.4)$ & Potassium lower agents & $3(3.6)$ \\
White & $68(81.9)$ & Polystyrene sulfonate & $1(1.2)$ \\
Other & $6(7.2)$ & Calcium polystyrene sulfonate & $1(1.2)$ \\
& & Potassium-binding agents & $1(1.2)$ \\
\hline Laboratory & Mean (SD, \%) & Glucose lowering therapies & $78(94.0)$ \\
eGFR CKD-EPI (serum creatinine) & $32.8(8.6)$ & Insulin & $48(57.8)$ \\
eGFR CKD-EPI (cystatin-C) & $37.8(8.8)$ & Metformin & $28(33.7)$ \\
Serum creatinine, mg/dL & $2.0(0.8)$ & Sulfonylurea & $22(26.5)$ \\
Cystatin C, mg/L & $1.8(0.5)$ & DPP-4 inhibitors & $13(15.7)$ \\
Phosphorus, mg/dL & $3.9(0.5)$ & GLP-1 agonists & $21(25.3)$ \\
Hemoglobin, g/dL & $12.6(1.7)$ & SGLT2 inhibitors & $7(8.4)$ \\
Hematocrit, \% & $37.5(4.8)$ & TZDs & $7(8.4)$ \\
UACR random, mg/g & $3,948.4(2,841.0)$ & Meglitinides & $1(1.2)$ \\
UACR 24 h, mg/g & $1,401.9(2,748.1)$ & Phosphate binders & $5(6.0)$ \\
Urea nitrogen, mg/dL & $39.0(16.0)$ & Antianemic preparations & $28(33.7)$ \\
Serum potassium, mEq/L & $4.7(0.5)$ & Iron preparations & $15(18.1)$ \\
Serum sodium, mEq/L & $139.9(2.6)$ & Erythropoietins & $3(3.6)$ \\
Serum bicarbonate, mEq/L & $20.4(3.2)$ & Other blood products & $3(3.6)$ \\
HbA1c & $7.2(1.0)$ & Vitamin B12 and folic acid & $12(14.5)$ \\
\hline
\end{tabular}

eFGR, estimated glomerular filtration rate; CKD, chronic kidney disease; ARB, angiotensin receptor blocker; ACEi, angiotensin-converting-enzyme inhibitor. 
Table 2. REACT for type 2 diabetes mellitus chronic DKD: clinical trial inclusion and exclusion criteria

\section{Inclusion criteria}

The participant is male or female, $30-80$ years of age on the date of informed consent

The participant has an established diagnosis of T2DM

The participant has an established diagnosis of diabetic nephropathy as the underlying cause of renal disease

The participant has an established diagnosis of CKD not requiring renal dialysis, defined as having an eGFR between 20 and $50 \mathrm{~mL} /$ $\mathrm{min} / 1.73 \mathrm{~m}^{2}$ inclusive at the screening visit and prior to REACT injection

The participant has blood pressure less than 150/90 at the screening visit, prior to renal biopsy, and prior to REACT injection(s). At the time of the biopsy and injections, the subject's BP should not be significantly below the previously recorded stable pressure

The participant has stable blood pressure and is maintained on a stable anti-hypertensive medication regimen if treatment for hypertension is necessary. If treatment includes an ACEi or an angiotensin receptor blocker, that treatment must have been initiated at least 8 weeks prior to renal biopsy. Treatment must be stable during the 6-week period immediately prior to REACT injection. Stable treatment is defined as dose adjustment to no less than one half of the current dosage or to no more than 2 times the current dosage. Dose interruptions up to 7 days due to medical necessity are allowed

A minimum of 2 measurements of eGFR or serum creatinine should be obtained at least 3 months apart prior to the screening visit or within the previous 18 months to define the rate of progression of CKD. The subject should have adequate historical clinical data to provide a reasonable estimate of the rate of progression of CKD. The medical monitor may be consulted to ensure that there are sufficient data

The participant is willing and able to refrain from NSAID consumption (including aspirin) as well as clopidogrel, prasugrel, or other platelet inhibitors during the period beginning 7 days before through 7 days after both the renal biopsy and REACT injection(s)

The participant is willing and able to refrain from consumption of fish oil and platelet aggregation inhibitors, such as dipyridamole (i.e., Persantine ${ }^{\circ}$ ), during the period beginning 7 days before through 7 days after both the renal biopsy and REACT injection(s)

The participant is willing and able to cooperate with all aspects of the protocol

The participant is willing and able to provide signed informed consent

\section{Exclusion criteria}

The participant has a history of type 1 diabetes mellitus

The participant has a history of renal transplantation

The participant has a serum $\mathrm{HbA} 1 \mathrm{c}$ level greater than $10 \%$ at the screening visit

The participant has uncontrolled diabetes (defined as metabolically unstable by the investigator)

The participant has abnormal coagulation status as measured by APTT, PT-INR, and/or platelet count at the screening visit

The participant has a bleeding disorder(s) or is taking anticoagulants, such as warfarin or direct thrombin inhibitors that, in the judgment of the investigator, would interfere with the performance of study procedures

The participant has small kidneys (average size less than $9 \mathrm{~cm}$ ) or has only one kidney, as assessed by ultrasound and/or MRI prior to renal biopsy, unless earlier radiology reports (generated within 1 year of the screening visit) are made available to confirm kidney size and number

The participant has known allergy(ies) or contraindication(s) or has experienced severe systemic reaction(s) to kanamycin or structurally similar aminoglycoside antibiotic(s)

The participant has a history of anaphylactic or severe systemic reaction(s) or contraindication(s) to human blood products or materials of animal origin (e.g., bovine and porcine)

The participant is not a good candidate to undergo percutaneous REACT injection, in the judgment of the surgeon or physician who will perform the procedure. This includes individuals who are morbidly obese (defined as BMI greater than $45 \mathrm{~kg} / \mathrm{m}^{2}$ ), have excessive fat surrounding the kidney, or who are otherwise at excessive risk for serious complications

The participant has a history of severe systemic reaction(s) or any contraindication to local anesthetics or sedatives

The participant has a clinically significant infection requiring parenteral antibiotics within 6 weeks of REACT injection

Renal Autologous Cell Therapy for Type 2

$\mathrm{DKD}$ 
Table 2 (continued)

The participant has acute kidney injury or has experienced a rapid decline in renal function during the last 3 months prior to REACT injection

The participant has any of the following conditions prior to REACT injection: renal tumors, polycystic kidney disease, anatomic abnormalities that would interfere with the REACT injection procedure, or evidence of a urinary tract infection

Note: Anatomic abnormalities are not exclusionary if the kidney remains accessible and meets the criteria to receive the REACT injection The participant has incapacitating cardiac and/or pulmonary disorders

The participant has a history of cancer within the past 3 years (excluding non-melanoma skin cancer and carcinoma in situ of the cervix)

The participant has clinically significant hepatic disease (ALT or AST greater than 3-times the upper limit of normal) as assessed at the screening visit

The participant is positive for active infection with HBV, or HCV, and/or HIV as assessed at the screening visit

The participant has a history of active TB requiring treatment within the past 3 years

The participant is immunocompromized or is receiving immunosuppressive agents, including individuals treated for chronic glomerulonephritis within 3 months of REACT injection

Note: Inhaled corticosteroids and chronic low-dose corticosteroids ( $\leq 7.5 \mathrm{mg}$ per day) are permitted as are brief pulsed corticosteroids for intermittent symptoms (e.g., asthma)

The participant has a life expectancy less than 2 years

The female participant is pregnant, lactating (breast feeding), or planning a pregnancy during the study. Or the female participant is of child-bearing potential and is not using a highly effective method(s) of birth control, including sexual abstinence. Or the female participant is unwilling to continue using a highly effective method of birth control throughout the duration of the study

Note: A highly effective method of birth control is defined as one that results in a low failure rate (i.e., less than one percent per year) when used consistently and correctly, such as implants, injectables, combined oral contraceptives, some IUDs, sexual abstinence, or a vasectomized partner

The participant has a history of active alcohol and/or drug abuse that, in the judgment of the investigator, would impair the subject's ability to comply with the protocol

The participant's health status would, in the judgment of the investigator, be jeopardized by participating in the study

The participant has used an investigational product within 3 months prior to REACT injection without receiving written consent from the medical monitor

REACT, Renal Autologous Cell Therapy; ACEi, angiotensin-converting-enzyme inhibitor; ARB, angiotensin receptor blocker; PTINR,prothrombin time-international normalized ratio; DKD, diabetic kidney disease; CKD, chronic kidney disease; eFGR, estimated glomerular filtration rate; APTT, activated partial thromboplastin time; HIV, human immunodeficiency virus; HBV, hepatitis B virus; HCV, hepatitis C virus; TB, tuberculosis; IUDs, intrauterine devices.

sent to the GMP facility for processing. An additional biopsy for local site histopathologic evaluation is allowed at the discretion of the investigator after obtaining the required cores for REACT manufacturing, depending on benefit versus risk of bleeding complications.

Selective Epithelial Renal Cell Isolation and Expansion: The cells are isolated by enzymatic digestion and expanded ex vivo, using standard cell culture techniques over approximately 4 weeks. SRCs are selected by density gradient centrifugation and formulated to produce REACT. REACT is shipped overnight as a thermolabile gelatin-based hydrogel fresh product to the clinical site, to be administered within $72 \mathrm{~h}$ from manufacturing release. RE$\mathrm{ACT}$ is warmed to room temperature for $30 \mathrm{~min}$ before the imageguided percutaneous injection into the patients' renal cortex of the same donor kidney, typically targeting a lower pole region. A 20-gauge outer guide needle (COOK Inc, Bloomington, IN, USA) is inserted into the subcapsular kidney, followed by co-axial insertion of an inner noncutting pencil tip 25-gauge needle (IMD, Huntsville, UT, USA) into the renal cortex within $5 \mathrm{~mm}$ of the renal capsule as shown in Figure 2. The injection target is a few millimeters deep to the subcapsular region to maximize cell deposition within the cortex microenvironment of the REACT source. The procedure is performed in an outpatient setting and with moderate sedation. Proceduralists undergo training, certification, and onsite proctoring.

The dose of REACT is $3 \times 10^{6}$ cells/g estimated kidney weight by volumetric imaging analysis from a noncontrast MRI scan of the kidneys, obtained during patient screening. An individualized dose volume is predetermined and divided into multiple REACT deposits into the kidney. We conduct real-time assessment of nee-
Stavas et al. 


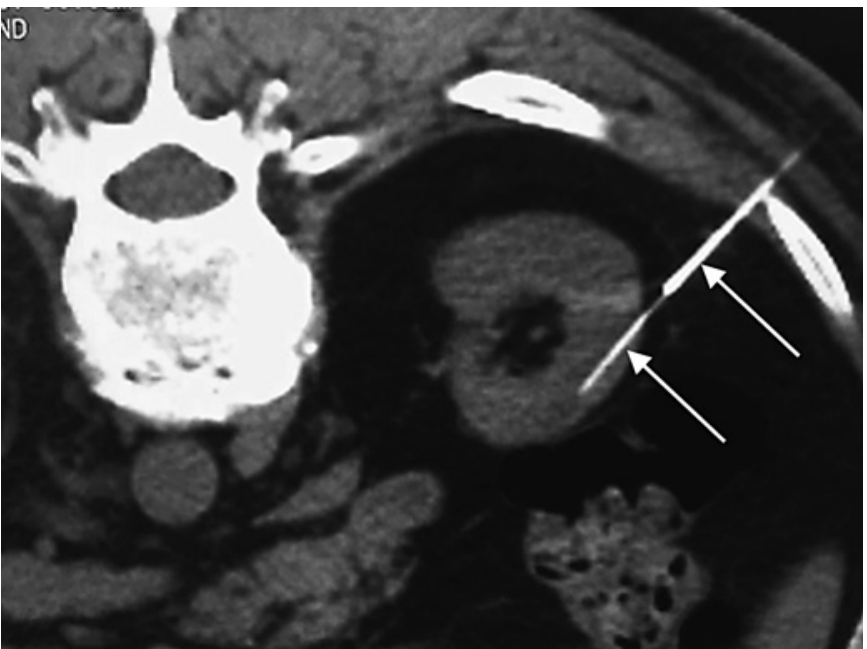

Fig. 2. Percutaneous CT-guided REACT injection. Patient in prone position in CT scanner. Insertion of outer guide and inner injection needles into the renal cortex in subcapsular location (arrows). CT allows real-time evaluation of needle location, REACT deposit, and bleeding complications. REACT, renal autologous cell therapy; CT, computed tomography.

dle location and REACT deposition, and determine any perinephric bleeding during intermittent CT scanning. Post-REACT recovery care is per local site, and it includes laboratory tests and delayed renal ultrasound to assess any procedure-related complication (i.e., renal hematoma). A 24-h follow-up clinical assessment, laboratory tests, and renal ultrasound are performed to determine renal function or delayed subclinical perinephric hematoma formation. A second REACT injection occurs approximately 6 months later into the same kidney.

Serial serum creatinine measurements are collected prerandomization and at 3-month intervals through 24 months after the last REACT injection, to calculate the eGFR by the CKD-EPI equation with serum creatinine. The rate of CKD progression for both the ATG and DTG will be compared. Additionally, each patient's disease progression rate pre-injection (eGFR slope derived from adequate historical clinical data) will be compared against the longitudinal rate of renal function decline, through the 24 months after the final REACT injection.

\section{Randomization}

We utilized a 1:1 randomization into 2 groups (ATG $n=42$ or DTG $n=41$ ) after manufacturing confirms the adequacy/quality of the donor kidney biopsy material and the date of REACT injection. The site investigator randomly assigned participants to either the ATG or the DTG using the Interactive Web Randomization System. Since this is an open-label study, the study participants, investigators, site staff, and sponsor are unblinded to the treatment assignment.

\section{Power}

Eighty-three patients who satisfied all the inclusion criteria were randomized 1:1 into either treatment group. The sample size was calculated based upon the phase I trial using an $80 \%$ power with an $\alpha=0.05$, assuming a $33 \%$ dropout rate to detect a difference of $50 \%$ in the eGFR between groups, as the primary efficacy endpoint. The trial size also allows sufficient safety information from the procedures and REACT product to be collected.

\section{Statistical Analysis}

Utilizing eGFR slope, standard error, and sample size for each study arm, a two-sample Walsh $t$ test will compare renal function and disease progression by CKD-EPI eGFR (2009), serum Cr, cystatin $\mathrm{C}, \mathrm{BUN}$, and urinary albumin at prerandomization, and through 24 months post-REACT injection. Patients will serve as their own control. The significance level of $\alpha=0.05$ will be set. We will evaluate the mean and standard deviation of the eGFR slope over time and at each endpoint, using a $t$ test to determine the difference between the ATG and the DTG. Values for missing data will not be imputed. $\chi^{2}$ analysis will compare the number of patients who experience AEs in both study groups.

The complete analysis set will include all patients enrolled in the study. The injection analysis set will include all patients who receive at least 1 REACT injection. Subgroup analyses will be performed to compare the safety and efficacy data from patients who received only a single REACT injection (e.g., due to exclusion criteria occurrences, dropout, death, or other reasons) versus patients who receive 2 REACT injections. Patient disposition (including screen failure, enrolled, successful biopsy, group assignment, number of REACT injections, withdrawn pre-injection or post-injection reasons, lost to follow-up, or completed study) will be summarized for the full analysis set by frequency and the proportion of patients.

Clinical and laboratory AEs will be coded using the Medical Dictionary for Regulatory Activities System Organ Class and Preferred Term, and we will present this information summarized. Treatmentemergent AEs by seriousness, intensity, the site investigator's assessment of relationship to the kidney biopsy, REACT injection procedure or REACT product, patient discontinuation due to AEs, or deaths will be included. The number of events (occurrence) and the number of participants (incidence) who experienced AEs will be reported. The number and percent of patients who develop clinically significant laboratory abnormalities longitudinally or exhibit changes on their physical examinations will be summarized.

\section{Clinical Outcomes}

The primary objective is to assess the safety and efficacy of up to 2 percutaneous REACT injections delivered 6 months $(+4$ weeks) apart, into the donor kidney. The primary safety endpoint is to assess procedure- and REACT product-related adverse events through 24 months after the last REACT injection, and the primary efficacy endpoint is the measurement of eGFRs from prerandomization through 24 months after the last REACT dose. Renalspecific AEs will be monitored. Other laboratory parameters, including urinary albumin, serum BUN, and urine microalbumin/ creatinine and protein/creatine ratios, were obtained prerandomization and through 24 months after the last REACT injection. Noncontrast MRI and renal scintigraphy imaging are performed during the trial to assess morphologic change and analysis of split renal function between treated and nontreated kidneys.

\section{Patient-Reported Outcomes}

Quality of life and patient satisfaction surveys are used as subjective patient assessments. Patient-reported outcomes from the kidney disease quality of life (KDQOL) and EQ-5D-5L surveys 
were obtained at baseline (defined as after randomization and before REACT injection) and through 24 months after the last REACT injection. A two-sample $t$ test will compare mean KDQOL and EQ-5D-5L scores between groups using a significance level of $\alpha=0.05$. Each patient's baseline KDQOL and EQ-5D- 5L scores will be compared against the individual patient's scores obtained through 24 months after the last REACT injection.

\section{Data Safety and Monitoring}

An independent Data and Safety Monitoring Board (DSMB) has been chartered to oversee patient safety, especially related to unexpected investigational product-related events. The DSMB consists of 3 members with expertise directly related to protocolspecified activities. It functions independently, and its members have no other engagement with the study sponsor. The DSMB meets at regular intervals depending on the rate of patient enrollment and new data generated, and advises the sponsor on aspects concerning the safety of patients participating in the clinical trial, specifically before the patients in the DTG receive their initial REACT injection. The DSMB reviews all safety and efficacy data obtained from interim analysis and may advise on dosing plans, protocol-specified evaluations, and follow-up procedures. The DSMB shares its recommendations with the study centers, institutional review boards/ethics committees, and regulatory authorities, as appropriate. Details of specific activities and responsibilities of the DSMB are in the DSMB charter.

\section{Discussion}

T2DKD imparts a large global burden on healthcare systems and economic costs to society. Diabetes may affect $25-28 \%$ of the US population by 2050 , with $>40 \%$ developing $\mathrm{CKD}$, resulting in high-cost comorbidities including end-stage kidney disease and renal replacement therapy $[5,14,15]$. Since the advent of renin-angiotensin system inhibitors, multiple classes of small molecule drugs have entered the clinical development pipeline to reduce DKD comorbidities, while targeting biochemical pathways that modulate metabolic, anti-inflammatory, or hemodynamic responses $[6,16,17]$. Nephroprotective effects of recent DKD therapies have demonstrated modest early reductions in declining eGFRs, although none impart improvement in the structural repair of the nephron. In addition, small molecule drugs are usually ineffective in advanced stages of DKD. Our phase II multicenter clinical trial of the novel intervention REACT will assess the safety and efficacy of autologous, homologous cell therapy in T2DKD and attempt to demonstrate nephrorestorative improvement in renal function.

Our preclinical studies with experimental models of CKD including DKD-induced and nephrectomy CKD models, showed increased survival, augmented renal function, and improved comorbidities after the cortex in- jection of SRCs, the active biological ingredient in REACT $[18,19]$. Preclinical cell markers identified an admixture of 3 cell sources (cap mesenchyme, ureteric bud, and podocyte), while lower levels of serum creatinine, blood urea nitrogen, and serum protein improved filtration correlating to a subpopulation of glomerular epithelial cells in SRCs. In addition, decreased glomerular sclerosis and mesangial proliferation in SRC-treated diabetic ZSF1 rodents were identified during necropsy at multiple interim analyses and may be predictive of positive cellular effects on multiple renal tissues in human DKD [12]. Ongoing evaluation of glomerular epithelial dysfunction in DKD may further define podocyte phenotypes that support the potential use of cellular therapies targeting renal function and proteinuria in CKD [20,21].

Cell integration into areas of renal inflammation and fibrosis was shown by in vivo confirmation of attenuated NF-kB and PAI-1 responses to monocytic and macrophage infiltration and upregulation of tubular expansion via trophic cues [22]. Local paracrine effects by exosome secretion of cytokines modulating repair of diabetes related damage from microvesicles may also have participated in the mechanism that resulted in observed antifibrosis and anti-inflammatory effects, and improved renal function [12].

Cell bioactivity and biodistribution of the SRCs were confirmed by injecting the labeled active biological product into the lower or upper renal pole regions of large and small animals [12, 23]. Superparamagnetic iron oxide contrast MRI and high-content image-based immunefluorescent analysis identified cellular dispersion throughout the kidney after locoregional injections, suggesting global implantation of cells into multiple nephrons. Gate signaling cytokines were identified in the cell mixture that elicit a chemotaxis response and mediate migration. In addition, the SRCs were identified in repaired glomeruli and tubules at necropsy utilizing immunohistology/histochemical methods and morphometric analysis $[12,23]$.

The SRCs of REACT biodistribution, improvement in renal function, reduced DKD comorbidities, and nonimmunogenic properties of selected renal autologous cells supported a phase I trial design. The phase I trial demonstrated feasibility and safety using hand-assisted laparoscopic access to the kidney under general anesthesia. Seven male patients underwent laparoscopic-guided cell injections with direct visualization using a large gauge needle [13]. Screening serum creatine was stable and unchanged to 18 months and increased at 24 months postinjection in 5 of 7 patients. In addition, renal clearance (calculated by Iohexol) and ACR were unchanged at 12 and 
24 months, respectively, whereas cystatin C eGFR was lower at 12 and 24 months than screening levels.

Nine serious postprocedure adverse events were determined to be related to the surgical procedures with general anesthesia in the Phase I study. No conclusive adverse events were associated with the cell-based product [13].

In the present phase II clinical trial, we converted the laparoscopic injection to a CT-guided percutaneous approach, given the advances in minimally invasive procedures with smaller-platform medical devices, imaging methods, and conscious sedation in an outpatient setting. These changes will mitigate adverse procedural events, and image guidance will ensure REACT enters the renal cortex. This approach currently is also used in a phase I open label trial of CKD due to congenital anomalies of the urinary tract [24].

\section{Conclusions}

REACT holds the promise to achieve improved renal function by nephron-repair and restoration of the diseased kidney with its unique cell composition and delivery method. The anti-fibrotic, anti-inflammatory, and nonimmunologic features of REACT may ameliorate the progression of DKD and reduce the need for renal replacement therapy. An improved structural function could complement and enrich the bioactivity of other small molecules affecting T2DKD renal function and comorbidities.

\section{Acknowledgements}

We wish to thank our REACT trial participants for their generous support of CKD research. We also would like to thank Ms. Brenda McGrath for statistical management and analysis, Ms. Chelsey Hehl for data administration assistance, and Ms. Beth Hilburger for manuscript preparation, all from ProKidney.

\section{Statement of Ethics}

The trial protocol was approved by each site's institutional review board or ethics committee on human research, and the participants provided written informed consent. The trial identifier number is NCT:02836574 and found at https:/clinicaltrials.gov/ ct2/show/NCT02836574.

\section{Conflict of Interest Statement}

J. Stavas, A. Johns, D. Jain, and T. Bertram are employed by ProKidney. S.G. Coca has received fees for advisory boards or steering committee roles for Renalytix, CHF Solutions, Bayer, Boehringer Ingelheim, Takeda, Vifor, Quark, ProKidney, and Akebia in the past 3 years. He owns equity in Renalytix, and receives salary and research support from Renalytix, ProKidney, XORTX, and the Renal Research Institute. A. Silva and M. Díaz-González de Ferris received fees for advisory board roles for ProKidney.

\section{Funding Source}

ProKidney, Grand Cayman, Cayman Islands, provided funding for the trial and manuscript submission.

\section{Author Contributions}

J.S. and M.F. made substantial contributions to the design of the work, drafting, revising, and final approval of the version to be published and accountable for all aspects of the work in ensuring that questions related to the accuracy or integrity of any part of the work are appropriately investigated and resolved. A.S., D.G., S.C., A.J., D.J., and T.B. contributed to revisions and final approval of the version to be published. G.B. contributed to trial design and final approval.

\section{Data Availability Statement}

Current trial safety and efficacy data are not publicly available due to active status of the trial.

\section{References}

1 Trounson A, McDonald C. Stem cell therapies in clinical trials: progress and challenges. Cell Stem Cell. 2015 Jul 2;17(1):11-22.

2 Farini A, Sitzia C, Erratico S, Meregalli M, Torrente Y. Clinical applications of mesenchymal stem cells in chronic diseases. Stem Cells Int. 2014;2014:306573.

3 Umanath K, Lewis JB. Update on diabetic nephropathy: core curriculum 2018. Am J Kidney Dis. 2018;71:884-95.

4 Molitch ME, Adler AI, Flyvbjerg A, Nelson RG, So WY, Wanner C, et al. Diabetic kidney disease: a clinical update from kidney disease: improving global outcomes. Kidney Int. 2015; 87:20-30.

5 Webster AC, Nagler EV, Morton RL, Masson P. Chronic kidney disease. Lancet. 2017;389: 1238-52.

6 Alici R, Johnson E, Tuttle K. SGLT2 inhibition for the prevention and treatment of diabetic kidney disease: a review. AJKD. 2018; 72(2):267-77.

7 Perkovic V, Jardine MJ, Neal B, Bompoint S, Heerspink HJL, Charytan DM, et al. Cana- gliflozin and renal outcomes in type 2 diabetes and nephropathy. N Engl J Med. 2019; 380(24):2295-306.

8 Lewis EJ, Hunsicker LG, Bain RP, Rohde RD. The effect of angiotensin-converting-enzyme inhibition on diabetic nephropathy. The Collaborative Study Group. N Engl J Med. 1993; 329:1456-62.

9 Marcheque J, Bussolati B, Csete M, Perin L. Concise reviews: stem cell and kidney regeneration. Stem Cells Transl. Med.. 2019;8:8292. 
10 Packman D, Fraser I, Kerr P, Segal K. Allogenic mesenchymal precursor cells (MPC) in diabetic nephropathy. A randomized, placebo-controlled, dose escalation study. EBioMedicine. 2016;12:263-9.

11 Benigni A, Morigi M, Remuzzi G. Kidney regeneration. The Lancet. 2010;375(9722): 1310-7.

12 Kelley R, Bruce A, Spencer T, Werdin E, Ilagan $\mathrm{R}$, Choudhury S, et al. A population of selected renal cells augments renal function and extends survival in the ZSF1 model of progressive diabetic nephropathy. Cell Transpl. 2013;22:1023-39.

13 Stenvinkel P, Wadström J, Bertram T, Detwiler R, Gerber D, Brismar TB, et al. Implantation of autologous selected renal cells in diabetic chronic kidney disease stages 3 and 4-clinical experience of a "First in Human" Study. Kidney Int Rep. 2016;1:105-13.

14 Boyle JP, Thompson TJ, Gregg EW, Barker LE, Williamson DF. Projection of the year 2050 burden of diabetes in the US adult population: dynamic modeling of incidence, mortality, and prediabetes prevalence. Popul Health Metr. 2010;8:29-2.

15 De Boer IH, Rue TC, Hall YN, Heagerty PJ, Weiss NS, Himmelfarb J. Temporal trends in the prevalence of diabetic kidney disease in the United States. JAMA. 2011;305(4):25329.

16 Heerspink HJL, Parving HH, Andress DL, Bakris G, Correa-Rotter R, Hou FF, et al. Atrasentan and renal events in patients with type 2 diabetes and chronic kidney disease (SONAR): a double-blind, randomized, placebo-controlled trial. Lancet. 2019;393:193747.

17 Bakris GL, Agarwal R, Anker SD, Pitt B, Ruilope LM, Rossing P, et al. Effect of finerenone on chronic kidney disease outcomes in type 2 diabetes. N Engl J Med. 2020;383(23): 2219-29.

18 Kelley R, Werdin ES, Bruce AT, Choudhury S, Wallace SM, Ilagan RM, et al. Tubular cellenriched subpopulation of primary renal cells improves survival and augments kidney function in rodent model of chronic kidney disease. Am J Physiol Ren Physiol. 2010;299(5): F1026-39.

19 Presnell SC, Bruce AT, Wallace SM, Choudhury S, Genheimer CW, Cox B, et al. Isolation, characterization, and expansion (ICE) methods for defined primary renal cell populations from rodent, canine, and human normal and diseased kidneys.
Tissue Eng C: Methods. 2011;17(3):26173.

20 Kawachi H, Fukusumi Y. New insight into podocyte slit diaphragm, a therapeutic target of proteinuria. Clin Exp Nephrol. 2020;24: 193-204.

21 Sagrinati C, Netti GS, Mazzinghi B, Lazzeri E, Liotta F, Frosali F, et al. Isolation and characterization of multipotent progenitor cells from the Bowman's capsule of adult human kidneys. J Am Soc Nephrol. 2006;17:2443-56.

22 Bruce AT, Ilagan RM, Guthrie KI, Rivera E, Choudhury S, Sangha N, et al. Selected renal cells modulate disease progression in rodent models of chronic kidney disease via NF- $\kappa \mathrm{B}$ and TGF- $\beta 1$ pathways. Regen Med. 2015; 10(7):815-39.

23 Basu J, Genheimer CW, O’Reilly D, Sangha N, Guthrie K, Bertram TA, et al. Distribution and analysis of stem and progenitor populations in large mammal and human kidneys. FASEB J. 2010 Apr;24(Suppl 1):lb35.

24 Stavas J, Ferris M, Johns A, Jain D, Bertram T. Protocol and baseline data on renal autologous cell therapy injection in adults with chronic kidney disease secondary to congenital anomalies of the kidney and urinary tract. Blood Purif. 2021;50(4-5):678-83. 